\title{
Detecção de Mudanças Florestais em Séries Temporais Utilizando os Algoritmos Landtrendr: Estudo de Caso no Estado do Rio de Janeiro
}

\author{
Time Series Forest Change Detection Using Landtrendr: \\ Study of Case in the State of Rio de Janeiro
}

\author{
Rômulo Weckmüller ${ }^{1} \bowtie$ (iD), Raúl Sánchez Vicens ${ }^{2} \bowtie$ \\ 1 Universidade Federal Fluminense, weckmuller@gmail.com, \\ https://orcid.org/0000-0002-8599-7211. \\ 2Universidade Federal Fluminense, rsvicens@id.uff.br
}

\begin{abstract}
Resumo: O entendimento de como os sistemas terrestres evoluem é importante na busca de estratégia que otimizem a utilização dos recursos naturais e minimizem os impactos ambientais. O monitoramento das mudanças da cobertura vegetal e do uso da terra, através de técnicas de sensoriamento remoto tem sido fundamental neste sentido. $\mathrm{O}$ objetivo principal deste artigo foi contribuir metodologicamente para o monitoramento dos sistemas terrestres através de técnicas de sensoriamento remoto, utilizando todo o acervo disponível de imagens Landsat, numa abordagem com base na trajetória espectral do pixel em séries temporais. Para tal, a detecção de mudanças baseada em trajetórias foi utilizada através do algoritmo LandTrendr, para detectar mudanças nas coberturas florestais do estado do Rio de Janeiro entre 1984 e 2016, identificando diferentes tipos de perturbações (desmatamentos e recuperações). Observou-se 58.969 ha de trajetórias de mudanças florestais no estado do Rio de Janeiro, destas 64\% são perdas florestais e $36 \%$ regenerações. Este mapeamento alcançou $70 \%$ de exatidão global. No litoral, as supressões são antigas e mais abruptas enquanto que no interior são antigas, porém graduais, configurando uma constante degradação da paisagem. As recuperações florestais no estado são mais recorrentes na Região Turística dos Lagos. Foi desenvolvido um mapa síntese com as características das trajetórias. Apenas esta região tem tendências de recuperação florestal, enquanto que as regiões no interior do estado têm tendências de degradação e as próximas ao litoral tendências de estabilidade, com taxas de ganho e perda florestal muito parecidas.
\end{abstract}

Palavras-chave: sensoriamento remoto, vegetação, Landsat.

Abstract: Understanding how terrestrial systems evolve is important in pursuing strategies that optimize the use of natural resources and minimize environmental impacts. Monitoring vegetation cover and land use changes through remote sensing techniques has been crucial in this regard. The main objective of this paper was to contribute methodologically to the monitoring of terrestrial systems through remote sensing techniques, using all the available collection of Landsat images, in an approach based on time series. For this trajectory-based change detection was used through the LandTrendr algorithm to detect changes in forest cover in Rio de Janeiro's state between 1984 and 2016, identifying different types of disturbances (deforestation and recovery) and classifying age of secondary forests. In a general way observed of 58969 hectares of forest changes in the state of Rio de Janeiro, these are 64\% of deforestation and 36\% of regenerations. This map achieved $70 \%$ global accuracy. On the coast, deforestation is old and more abrupt, while inland forest losses are also old but gradual, creating a constant degradation of the landscape. Forest recoveries in the Rio de Janeiro's state are more recurrent in Lagos Tourist region. A synthesis map was developed with trajectory characteristics. Only the Lagos region has forest recovery tendencies, whereas the regions in the interior of the state have tendencies of degradation. The regions near the coastal have tendencies of stability which rates of gain and loss very similar.

Key words: remote sensing, vegetation, Landsat. 


\section{Introdução}

Estudos de mudanças na cobertura da terra são importantes, pois seus resultados são fundamentais para ações de planejamento e/ou gestão do território. Eles contemplam a quantificação e localização de supressões, urbanizações, regenerações, entre outros. Tais informações têm como suporte o processamento de imagens multitemporais e detecção de mudanças, que tem sido um campo de pesquisa ativa em sensoriamento remoto por décadas, tornando-o a maior fonte de dados espacializados atualmente para o monitoramento da cobertura da terra (LU et al., 2004; LU et al., 2014; JIANYA et al., 2008). As trajetórias evolutivas surgem como complemento dos estudos de mudanças, aprofundando as informações sobre o histórico da cobertura da terra. Esta perspectiva abre um horizonte maior de entendimento sobre a dinâmica da paisagem (WECKMÜLLER, 2018).

De acordo com Banskota et al. (2014), os métodos de detecção de mudança baseadas em trajetórias utilizam padrões espectrais-temporais para identificar tipos de distúrbios e suas magnitudes. Os autores as dividem em 4 categorias: (1) Baseada em limiares, (2) Simples ajuste da curva, (3) Curva hipotética e (4) segmentação da trajetória. A categoria 1 exige como pré-requisito um limiar que configure mudanças florestais. Já as categorias 2, 3 e 4 geram suas próprias trajetórias estimando os limiares com base nas informações da série temporal. Kennedy et al. (2014) classificaram os diferentes tipos de trajetórias de acordo com suas curvas espectrais-temporais: (a) estresse ou perda crônica; (b) ganho ou aumento; (c) mudança definitiva; (d) mudança e resiliência; (e) mudança cíclica.

Como as mudanças induzidas pelo homem ocorrem em um ritmo cada vez mais rápido, espera-se que os sistemas de monitoramento baseados em sensoriamento remoto, que imageam o Planeta Terra em tempo integral, tenham papéis cruciais na política ambiental e tomada de decisão (CHEN et al., 2012). A observação da Terra por satélites é a única maneira de providenciar uma contínua e consistente base de dados sobre a cobertura da terra. Esta abundância de dados temporais possibilita a obtenção das trajetórias evolutivas da cobertura da terra, que representam uma assinatura espectral-temporal da paisagem (MAUS et al., 2016). Assim, de acordo com Câmara et al. (2016), o mapa deixa de ser um resultado absoluto, exigindo o complemento de uma assinatura espectral-temporal de suas classes de mudança, seguindo o novo paradigma dos estudos de mudança, que prioriza a variável tempo, através de análise de séries temporais.

Câmara et al. (2016) defendem as trajetórias evolutivas obtidas de séries temporais como uma quebra de paradigma nos estudos de mudança. Até agora a variável espaço era priorizada em relação ao tempo, ou seja, a detecção de mudanças é feita para cada objeto espacial a partir da comparação em vários cenários temporais, é o paradigma chamado de "Space First". Recentemente tem aparecido uma mudança neste paradigma, pois a variável tempo passa a ocupar um lugar de destaque, com o uso de séries temporais e a detecção no tempo dos disturbios responsáveis pelas mudanças nos geo-objetos. Este novo paradigma tem sido chamado de "Time First" (CÂMARA et al., 2016). Desta maneira, o mapa deixa de ser um resultado absoluto, exigindo o complemento de uma assinatura espectral-temporal de suas classes de mudança (MAUS et al., 2016). Kennedy et al. (2014) concordam com este novo paradigma, na medida em que também defendem a utilização de séries temporais, pois acreditam que a bitemporalidade não é capaz de definir qual processo de trajetórias está atuante na paisagem.

A detecção de mudanças com base em trajetórias vem ganhando espaço com a disponibilização de longas séries temporais, a partir da modelagem das curvas de trajetórias e a classificação da curva de cada pixel. O algoritmo de detecção de mudanças com base em trajetória, desenvolvido por Kennedy et al. (2007) fornece estimativas tanto sobre desvios bruscos da trajetória (como a intensidade e a data da perturbação) quanto de processos contínuos e lentos (como a revegetação). O método baseia-se no melhor ajuste da curva temporal de cada pixel em relação a trajetórias reconhecidas numa área de floresta. Posteriormente, Kennedy et al. (2010) introduziram e testaram o algoritmo LandTrendr (Landsat-based detection of Trends in Disturbance and Recovery) para extrair trajetórias de mudanças na cobertura da terra a partir de series temporais Landsat.

Vários trabalhos sobre detecção de trajetórias têm sido publicados recentemente, utilizando o algoritmo LandTrendr. Kennedy et al. (2012) analisaram padrões de desmatamentos e recuperações vegetais em áreas protegidas públicas e privadas, além das ecorregiões de uma floresta temperada no noroeste dos Estados Unidos. Griffiths et al. (2012) adotaram as trajetórias evolutivas em uma cena Landsat que corresponde à parte da região dos Cárpatos, no leste da Romênia, para entender o comportamento da cobertura da terra nos principais acontecimentos na Europa entre 1984 e 2010, concluindo que o colapso do socialismo provocou uma diminuição drástica no desmatamento e o abandono de muitas terras agrícolas, enquanto que a ascensão da União Europeia contribuiu para a retomada de cultivos nestas áreas agrícolas. Fragal et al. (2016) utilizam as trajetórias para mensurar a perda de florestas de várzea num trecho do baixo rio Amazonas entre $1984 \mathrm{e}$ 2009. Os autores concluíram que o LandTrendr obteve uma acurácia entre 65 e $70 \%$ de acerto nas classes 
propostas. Kennedy et al. (2018) implementaram os algoritmos LandTrendr na plataforma Google Earth Engine, devido ao grande acervo de imagens e capacidade de processamento em nuvem deste. Os autores conseguiram entre $85 \%$ e $95 \%$ de concordância entre os resultados do algoritmo no Envi IDL e no software do Google.

Neste contexto, este trabalho objetiva mapear as trajetórias florestais (de supressão e recuperação) do estado do Rio de Janeiro, entre 1984 e 2016, utilizandos os algoritmos LandTrendr. Com o objetivo específico pretende-se analisar o ano inicial, a magnitude e a duração destas mudanças florestais, para entender seus padrões espaciais. Para recobrir a área de estudo são necessárias seis cenas do satélite Landsat (Figura 1). A escolha desta área justifica-se pelo alto dinamismo na cobertura da terra do estado nas últimas décadas, a carência de estudos desta natureza a nível estadual e a histórica pressão antrópica dos ciclos econômicos sobre a Mata Atlântica da região.

Originalmente toda a Serra do Mar no estado do Rio de Janeiro era recoberta pela Mata Atlântica, com um perfil de vegetação marcado pela transição das formações florestais em função da umidade e temperatura. Dentre as fitofisionomias de Mata Atlântica da região destaca-se a floresta ombrófila densa, presente em todo o litoral e nas serras, onde há uma regime regular de chuvas durante todo o ano. No extremo norte, com um clima bem mais seco, há um predomínio de floresta estacional decidual e floresta estacional semidecidual (VELOSO et al., 1991; VICENS et al., 2009).

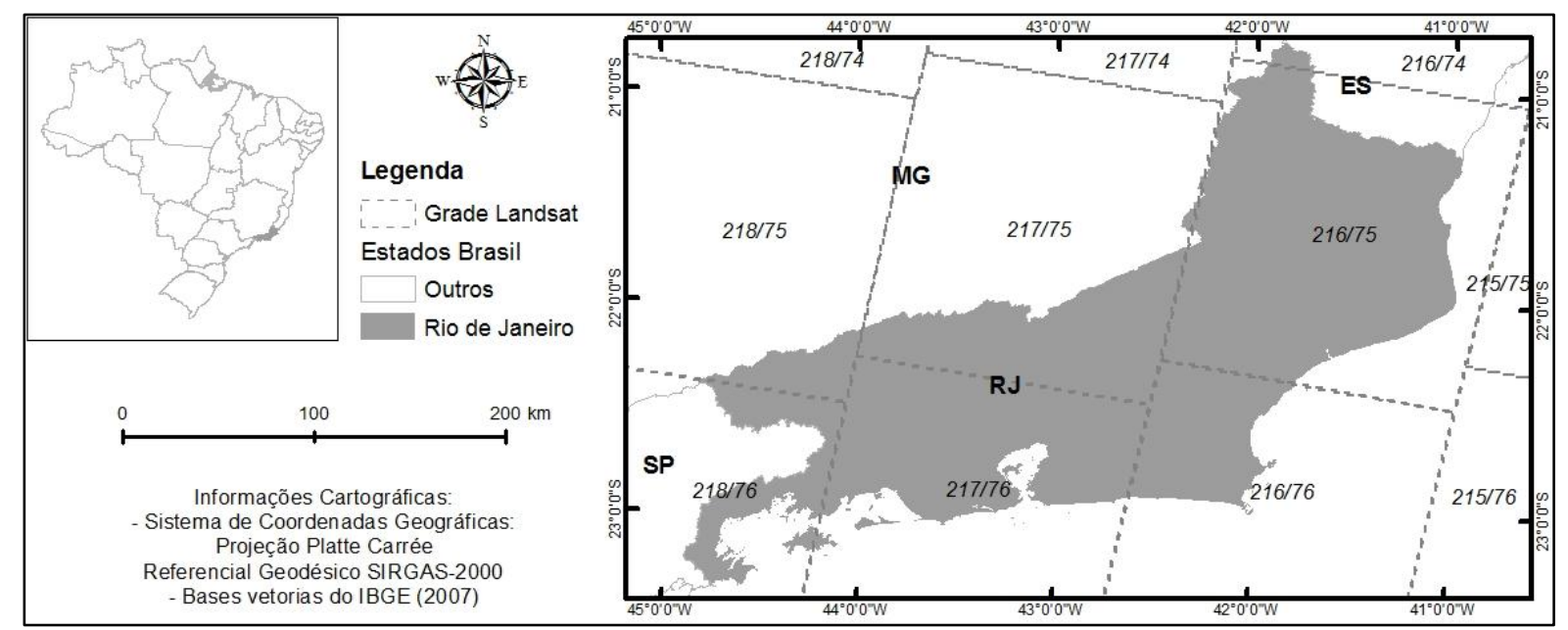

Figura 1: Cenas Landsat que cobrem o estado do Rio de Janeiro: órbitas 216, 217 e 218 com os pontos 075 e 076 .

Os 92 municípios pertencentes ao estado do Rio de Janeiro estão divididos em regiões de governo de acordo com suas características socioeconômicas. Estas regiões foram estabelecidas em 1987 e de acordo com Saraça et al. (2007) não retratam mais a realidade, pois as recentes mudanças na estrutura produtiva acarretaram em uma reordenação espacial que impôs a necessidade de se formular uma nova regionalização. Seguindo este raciocínio, a fundação CIDE (atual CEPERJ) propôs uma nova regionalização para o estado que levam em consideração as diferenças e particularidades mais atuais, baseada nas regiões de 1987, porém apresenta algumas modificações na forma de agrupamento dos municípios. Esta nova regionalização propõe a divisão do estado em 9 regiões e foi usada como base para nortear alguns resultados deste artigo (Figura 2). 


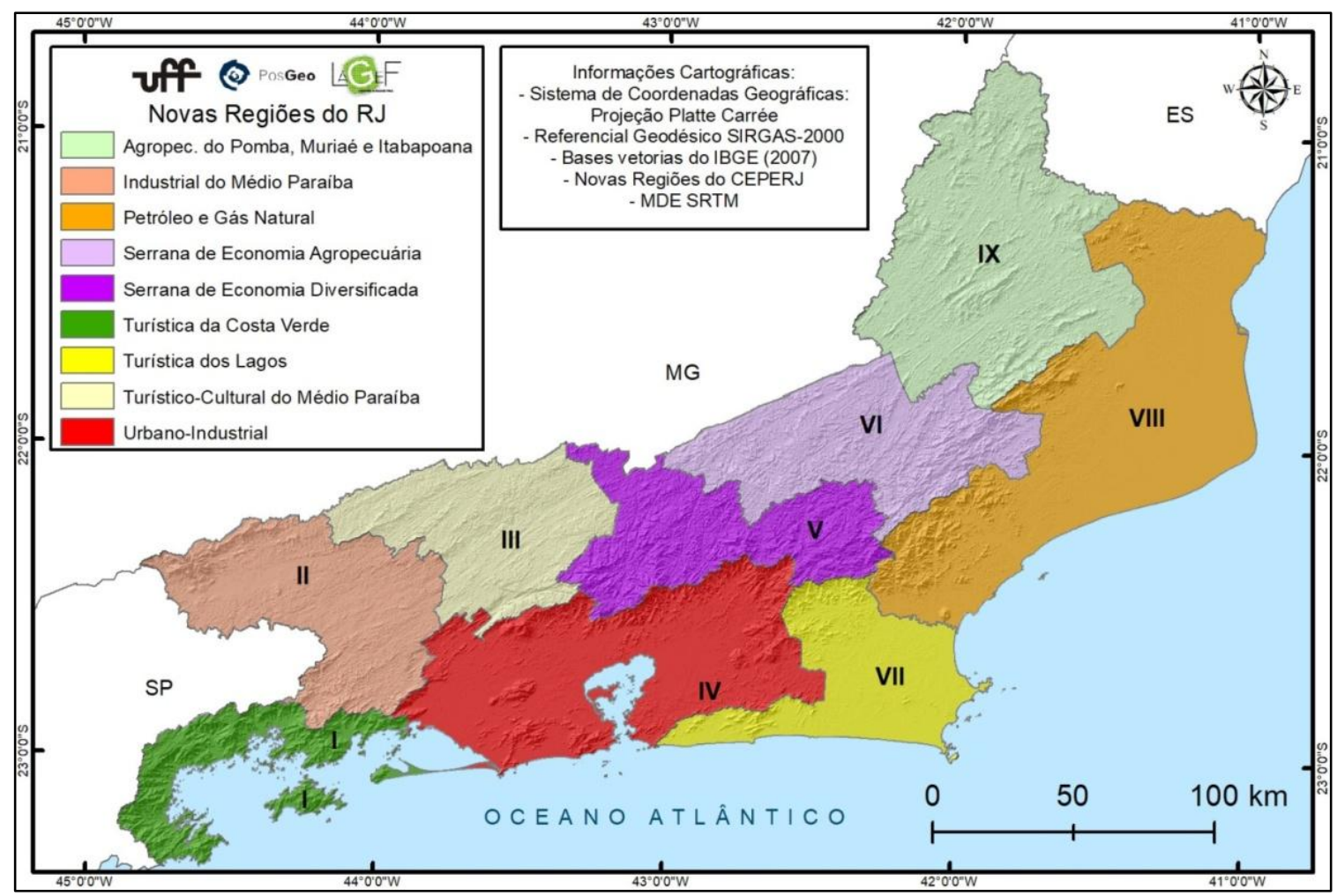

Figura 2: Novas regiões político-administrativas do Rio de Janeiro (proposta da CEPERJ).

\section{Materiais e Métodos}

\subsection{Materiais}

Para o presente trabalho foram utilizadas imagens Landsat, dos sensores TM (1984 a 2011) e OLI (2013 a 2016). Tais imagens são atualmente disponibilizadas gratuitamente pelo USGS (United States Geological Survey, portal: http://earthexplorer.usgs.gov/), na forma do produto Landsat Surface Reflectance Climate Data Record (CDR). O intervalo de imageamento foi anual, cerca de 32 imagens, para o período entre 1984 e 2016, para cada uma das seis cenas que compõe o estado, totalizando 178 imagens. Foram priorizadas imagens no período do outono e inverno, onde há na área de estudo uma menor incidência de nuvens e menos umidade na vegetação. Quando houve muita presença de nuvens nestas estações, imagens de outros períodos foram utilizadas. Para alguns anos não foram encontradas imagens com pouca cobertura de nuvens.

Para a classificação das trajetórias foi utilizado o algoritmo Landsat-based Detection of Trends in Disturbance and Recovery. O LandTrendr é um pacote de algoritmos de detecção de mudanças baseado em pixels, desenvolvido por Kennedy et al. (2010), que objetiva gerar trajetórias espectro-temporais que expressam os eventos ocorridos no pixel no decorrer do tempo, resumidamente em três fases: préprocessamento, segmentação temporal e classificação das trajetórias. Este pacote de algoritmos também possibilita a obtenção de características dos eventos florestais, como seu ano inicial, magnitude e duração do distúrbio. Além de estar disponível gratuitamente à comunidade científica no portal eletrônico da Universidade de Oregon (http://landtrendr.forestry.oregonstate.edu/), dispõe de um documento de guia do usuário que detalha bem toda a organização de pastas, processamento de dados de entrada e execução do algoritmo, no software Envi IDL. Em Kennedy et al. (2018), o LandTrendr foi adicionado ao Google Earth Engine (disponível gratuitamente em: https://github.com/KennedyResearch/LandTrendr-2012).

\subsection{Pré-Processamento}

As imagens Landsat do produto CDR obtidas no portal eletrônico do USGS, de acordo com seus metadados, já vem ortorretificadas com precisão sub-pixel, corrigidas segundo a atmosfera utilizando o modelo 6S. Porém numa detecção de mudanças, o mais importante seria a pouca variação das imagens entre si. Por isso optou-se pela realização de uma normalização radiométrica, objetivando diminuir a variação radiométrica dentro da série temporal (CRONEMBERGER, 2014; WECKMÜLLER;VICENS, 2016; FERNANDES et al., 2017). Importante destacar que no produto CDR há uma máscara de nuvem associada a cada imagem, evitando problemas de falsas mudanças causadas por presenças de nuvens na série. 
Além disso, para restringir a área de análise, foi desenvolvida uma máscara de floresta para limitar a interpretação dos dados gerados pelos algoritmos. Após testes preliminares com o LandTrendr, observou-se a necessidade de desenvolvimento de uma máscara de sombras topográficas, pois as mesmas eram classificadas erroneamente como mudança. Uma máscara de silvicultura foi adquirida para evitar falsas recuperações.

\subsubsection{Normalização radiométrica}

A normalização radiométrica foi realizada utilizando pontos pseudo invariantes (Pseudo-invariant Features - PIF) ao longo do tempo entre uma imagem de referência e a imagem a ser normalizada, a partir dos quais são calculados os parâmetros da função de regressão linear ordinária mínimos quadrados, seguindo o método utilizado por Schott et al (1988). Todo o processo foi programado em ambiente R (FERNANDES et al., 2017). O código normaliza em pares de imagens, desenvolvendo uma equação de regressão linear para cada ano da série temporal, levando-se em conta a mesma imagem referência. Os dados de saída são a imagem normalizada e um gráfico entre esta e a imagem referência para análise de qualidade dos resultados (Op. cit.).

\subsubsection{Desenvolvimento de máscaras}

Com o objetivo de limitar a área de estudo somente para as coberturas florestais do estado, uma máscara de floresta foi utilizada (WECKMÜLLER, 2018). O desenvolvimento desta foi por análise orientada a objetos, baseada na lógica fuzzy e hierarquia de classes, numa tentativa de "modelar o conhecimento" (CRUZ et al., 2007).

A máscara de sombreamento topográfico foi desenvolvida com base no processamento do MDE SRTM (30 metros), para a obtenção da radiação anual. Áreas com menos de 450 horas de luz solar por ano foram consideradas como máscara (WECKMÜLLER, 2018). Por fim, foi utilizada uma máscara de silvicultura, desenvolvida por Ferraz (2017), com o objetivo de diferenciar trajetórias de regeneração das trajetórias de florestas manejadas, que apresentam esse tipo de comportamento.

Como já mencionado, cada imagem na série tinha uma máscara de nuvem associada, obtidas através do produto CDR do USGS. Estas foram binarizadas, onde o pixel igual a 0 representa nuvem/sombra de nuvem e igual a 1 são as áreas sem nuvem (somente nestes o algoritmo foi executado e analisado na série temporal).

\subsection{LandTrendr}

\subsubsection{Segmentação temporal}

O conceito de segmentação temporal, é importante, pois se difere do uso da palavra segmentação usada até então na literatura, de cunho estritamente espacial. A segmentação temporal do algoritmo LandTrendr consiste em identificar quebras estatísticas do comportamento linear entre a imagem inicial e a final da série, simplificando a série espectro-temporal. Esta função linear entre o ano inicial e o final é quebrada quando há algum valor no meio da série com um desvio maior do que o limiar booleano definido pelo pesquisador como não mudança. Esta série ajustada pode ser relacionada a eventos ocorridos com a cobertura florestal, como supressões e regenerações. Ambos podem ser observados pelos segmentos das trajetórias, que mostram os momentos críticos de cada mudança (KENNEDY et al., 2010; WECKMÜLLER, 2018).

Por meio desses segmentos são gerados os atributos de duração, magnitude e ano de início da perda ou regeneração da cobertura florestal. Assim, o atributo de duração indica o tempo levado para a perda ou regeneração da floresta, o atributo de magnitude expressa a amplitude de variação do índice espectral, então, quanto maior a magnitude mais abrupta foi a perda da cobertura florestal e por fim, o ano de início expressa quando o evento de supressão ou regeneração teve início (FRAGAL et al., 2016).

Dos vários descritores disponíveis para o processo de segmentação temporal, Weckmüller et al. (2018) testaram cinco, a saber: banda 5, greenness, NBR, NDVI e wetness. A banda 5 (infravermelho médio) foi escolhida com base em Kennedy et al. (2007) e seu algoritmo mpfit (predecessor do LandTrendr), que apontou a importância desta faixa espectral para detecção de mudanças em floresta. O greenness representa o verdor da vegetação, quanto maior a biomassa mais brilhante é o pixel, por isso, tinha-se uma expectativa quanto ao seu desempenho. Já o NDVI, de acordo com Jensen (2009) e Ponzoni e Shimabukuro (2009), é um índice consagrado em estudos de vegetação, pois além de ser sensível a mudanças no dossel florestal, a razão entre bandas proporciona uma redução de muitas formas de ruídos, como algumas variações topográficas e 
de iluminação solar, por exemplo. O NBR, de acordo com Cohen et al. (2010), aproveita as vantagens entre a reflectância do infravermelho próximo e médio, sendo uma vantagem para mapear vegetações. Griffith et al. (2012) acrescentam que o NBR é um ótimo índice para detectar distúrbios em florestas temperadas. Já o Wetness, uma combinação linear de seis bandas espectrais Landsat, é um índice bem estabelecido nos estudos de trajetórias florestais realizados por Cohen et al. (2010).

Após testes estatísticos que mensuraram a performance dos descritores, que podem ser consultados em Weckmüller et al. (2018), os autores concluíram que o NDVI obteve o melhor desempenho na detecção de mudanças em florestas tropicais, caso da área de estudo. Resultados estes corroborados por Fragal (2015). Sabe-se que o NDVI tende a saturar em florestas densas, devido a sombreamentos causados pela heterogeneidade no dossel florestal. De acordo com Ponzoni e Shimabukuro (2009), esta característica seria uma limitação para o mapeamento de diferentes fitofisionomias, mas não para uma detecção de mudanças florestais.

Definido o descritor, resta configurar os parâmetros de controle para garantir uma segmentação temporal que contemple as trajetórias florestais da área. Os principais parâmetros que influenciam na qualidade da segmentação temporal são: kernel size, pval e max segments (KENNEDY et al., 2010). Após sucessivos testes em área de controle, respectivamente, definiu-se 3x3, 0.05 e 6 como os valores mais adequados para estes parâmetros, condizentes com os valores encontrados por Fragal (2015).

\subsubsection{Classificação das trajetórias florestais}

A fase de classificação é a última do processo de detecção de trajetórias. Neste momento são inseridos os parâmetros de controle de mudanças (limiares booleanos) e as classes que representam as trajetórias da paisagem, através do algoritmo chamado labelfit, que faz parte do pacote de algoritmos LandTrendr. O labelfit possui várias classes diferentes, que podem ser configuradas e adaptadas de acordo com as necessidades do pesquisador e/ou da área de estudo. Cada classe possui parâmetros espectro-temporais e de filtragem bem definidos, sendo imprescindível uma análise detalhada do guia do usuário do LandTrendr e experiência do pesquisador em estudos de séries temporais. As classes apresentam perdas e ganhos de vegetação em duas velocidades distintas, definida pelos autores: abrupta (menos de 5 anos) e gradual (mais de 5 anos).

Para definir os limiares de mudanças florestais, amostras previamente conhecidas na paisagem foram coletadas (de supressões e regenerações) e inseridas no software Weka para mineração de dados utilizando árvores de decisão através do algoritmo J48. Os limiares propostos pelo processo de mineração de dados associado ao uso de ferramentas do software Envi que permitem visualizar de forma dinâmica os valores espectrais da série permitiram definir os limiares utilizados neste trabalho: para uma mudança ser considerada supressão vegetal tem que apresentar perda anual maior ou igual a 0.22 e perda ao longo de 20 anos de série maior ou igual a 0.18 no NDVI; já para ser considerada uma recuperação da vegetação, basta ganho maior ou igual a 0.27 no NDVI.

Quanto à construção das classes, o LandTrendr possui em sua biblioteca 9 classes pré-definidas de trajetórias, numa linguagem de programação própria e disponível no seu manual, o que possibilita a total personalização destas. Para cada classe existem configurações de filtros diferentes associadas, de passa alta ou passa baixa, dependendo das características da classe. Estes também podem ser alterados de acordo com a necessidade do usuário.

De maneira resumida podemos classificar as classes de trajetórias do LandTrendr em 3 grupos: invariantes (classe 1 e 2), distúrbios ou desmatamentos (3 a 7) e recuperações ( 8 e 9). Basicamente elas se diferem por tempo de perturbação, existem recuperações rápidas e graduais, por exemplo. Optou-se também por manter os valores de filtragem originais, visto a falta de resultados significativos entre diferentes configurações testadas.

\subsubsection{Mapeamento das características das trajetórias}

Para cada classe do LandTrendr, o algoritmo gera um arquivo raster com 8 bandas, onde cada uma delas apresenta uma característica diferente sobre aquela classe de trajetória. As 3 primeiras bandas (ano inicial, duração e magnitude do evento) foram utilizadas e possibilitaram gerar mapas que auxiliaram na descrição e entendimento das trajetórias. Estes resultados também receberam aplicação das máscaras e precisaram ser adaptados conforme a legenda final, gerando assim os mapas de ano inicial, duração e magnitude dos desmatamentos e recuperações florestais no estado do Rio de Janeiro. 
O mapeamento de ano inicial permite obter a idade dos fenômenos observados. Já a magnitude expressa a inclinação do segmento de mudança. Quanto mais inclinado, mais abrupta foi a mudança, quanto menos inclinado, mais gradual. A duração contém a quantidade de tempo (em anos) que o fenômeno durou até a sua estabilização. A associação entre estes três mapeamentos permite entender o grau de degradação, estabilidade ou recuperação da paisagem.

\subsection{Validação}

A estratégia de validação foi a utilização do índice Kappa associado à análise da matriz de confusão. Para tal agrupou-se as trajetórias em três grandes grupos, a saber: florestas invariantes, trajetórias de supressão e trajetórias de regeneração. Foram gerados 450 pontos aleatórios para cada um dos três grupos mencionados, totalizando 1350 pontos (50 pontos para cada uma das 9 regiões do estado). Estes pontos foram analisados um a um tendo como referência a própria série temporal.

\subsection{Mapa Síntese das Trajetórias}

Com o objetivo de sintetizar as características de trajetórias florestais foi desenvolvido um mapa síntese, dividido por região do estado do Rio de Janeiro. Para tal, uma planilha com todos os dados produzidos pelo algoritmo foi montada para auxiliar a confecção deste mapa.

Esta planilha foi desenvolvida em 2 níveis. No primeiro nível tem-se a proporção de trajetórias de supressões e recuperações por região, detectando se a paisagem tem uma tendência de degradação, estabilidade ou recuperação. No segundo, os percentuais das classes de ano inicial, magnitude e duração dos desmatamentos e recuperações, devidamente simplificados para uma legenda nominal. Assim, caso no nível 1 a região tenha um predomínio de recuperações, as características destas e suas predominâncias são observadas no nível 2, dando origem a uma classificação final no mapa síntese. A Figura 3 resume as etapas metodológicas deste trabalho.

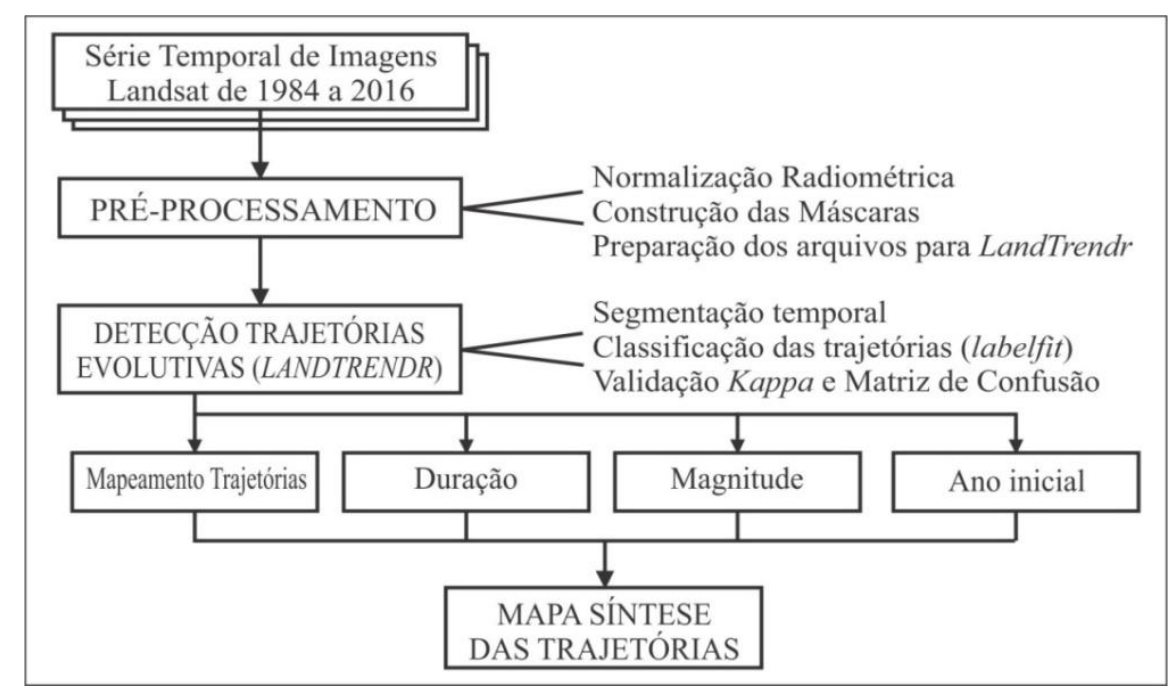

Figura 3: Etapas metodológicas resumidas do trabalho.

\section{Resultados e Discussão}

\subsection{Normalização radiométrica}

O código que automatizou a normalização radiométrica gerou gráficos para cada processo, contendo a reta de regressão linear entre a imagem referência e a imagem a ser normalizada, o coeficiente de determinação $\left(\mathrm{R}^{2}\right)$ e as equações antes e depois do processo de normalização. Estes gráficos possibilitaram avaliar a qualidade dos produtos gerados. Considerando que o processo é realizado em pares, numa série temporal com mais de 30 anos e em 6 cenas do satélite Landsat, fica inviável demonstrar aqui todos os gráficos gerados.

Para mensurar a qualidade das normalizações recomenda-se analisar o coeficiente de determinação $\left(\mathrm{R}^{2}\right)$, que corresponde à correlação que existe entre os PIFs coletados entre as imagens, e as equações de 
regressões lineares, que representam o quanto a imagem a ser normalizada se aproximou da imagem referência. Dada a grande heterogeneidade das equações, que de uma maneira geral aproximaram os dois eixos do gráfico (aproximando a imagem referência da normalizada), optou-se por generalizar os resultados obtidos no coeficiente de determinação $\left(\mathrm{R}^{2}\right)$ por banda Landsat (Tabela 1).

Tabela 1: Porcentagem de imagens por intervalo de valor do $\mathrm{R}^{2}$. Bandas espectrais: (B) azul; (G) verde; (R) vermelho; (NIR) infravermelho próximo; (SWIR1) infravermelho médio 1; (SWIR2) infravermelho médio 2.

\begin{tabular}{ccccc}
\hline \multirow{2}{*}{ Bandas } & \multicolumn{4}{c}{ \% de imagens normalizadas por intervalor de valor do $\mathbf{R}^{\mathbf{2}}$} \\
\cline { 2 - 5 } 1 (B) & Menor 0.5 & $0.5-0.69$ & $0.7-0.8$ & Maior 0.8 \\
\cline { 2 - 5 } 2 (G) & 44 & 36 & 12 & 7 \\
$3(\mathrm{R})$ & 37 & 50 & 12 & 3 \\
4 (NIR) & 29 & 45 & 23 & 78 \\
5 (SWIR 1) & 10 & 3 & 9 & 87 \\
7 (SWIR 2) & 2 & 6 & 6 & 62 \\
\hline
\end{tabular}

Houve uma maior correlação nas bandas do infravermelho (4, 5 e 7), com um $\mathrm{R}^{2}$ de 0.8 ou mais como predominante, em relação às bandas do visível $(1,2$ e 3$)$, que em sua maioria, obtiveram um $\mathrm{R}^{2}$ menor que 0.7; com um melhor desempenho da banda 3 (vermelho). Porém, como foi utilizado o NDVI no processo de segmentação temporal e o mesmo utiliza as bandas 3 e 4 na sua equação, acredita-se que mesmo este resultado um pouco inferior do vermelho não prejudicará a representatividade deste dado em relação à identificação da vegetação.

Para testar o quanto o processo de normalização aproximou os valores entre as bandas comparou-se as diferenças existentes entre os PIFs antes e depois da normalização. De uma maneira geral o processo conseguiu aproximar os valores das imagens em duas vezes, em média, variando para uma maior aproximação nas bandas infravermelhas e uma menor nas bandas do visível. Espera-se que essa aproximação entre as imagens resulte em uma menor incidência de falsas mudanças.

\subsection{Trajetórias florestais do estado do Rio de Janeiro}

Após o processo de segmentação temporal, classificação pelo labelfit, aplicação das máscaras e edição manual, foi gerado o mapa de trajetórias florestais. Os resultados mostraram que 58.969 ha de áreas florestais no estado do Rio de Janeiro, apresentaram algum tipo trajetória associada a mudanças na cobertura vegetal, detalhadas na Tabela 2. Estes quase 59000 ha de trajetórias representam aproximadamente $6 \%$ das florestas observadas em 1984, ou seja, nos 32 anos de análise o predomínio na área foi de invariância de florestas. Agrupando as classes de desmatamentos e de recuperações (Tabela 2), percebe-se que as trajetórias de perdas florestais correspondem a 34.419 ha (64\% do total da área que apresentam mudanças), enquanto que as de recuperações a 19.699 ha (36\%). Note que a classe "Recuperação (Silvicultura)" não foi computada no cálculo da proporção, pois a mesma não foi considerada como uma regeneração efetiva de áreas de floresta pelo seu comportamento cíclico, representando uma área de cultivo.

Tabela 2: Área em hectares das classes de trajetórias florestais do Rio de Janeiro.

\begin{tabular}{lcc}
\hline \multicolumn{1}{c}{ Classes de Trajetórias Florestais } & Área (ha) & Áreas (ha) agrupadas \\
\hline Florestas Invariantes & 1274600 & 4310697 \\
Outras coberturas & 3036097 & \\
Desmatamento Abrupto & 10905 & 34419 \\
Desmatamento Duplo & 185 & \\
Desmatamento Gradual & 23329 & 19699 \\
Desmatamento seguido de recuperação & 53 & \\
Recuperação Abrupta & 19341 & 4850 \\
Recuperação Gradual & 305 & \\
Recuperação (Silvicultura) & 4850 & \\
\hline
\end{tabular}


Com o objetivo de aprofundar a análise das trajetórias à procura de um padrão de localização, visto a característica fragmentada da mesma, foram desenvolvidos dois mapas de densidade kernel, um com as trajetórias de supressões e outro com as de recuperação (Figuras 4 e 5). Estes mapas apresentam as áreas onde cada um dos fenômenos foi predominante, porém foram usados apenas como recurso visual, visto sua imprecisão cartográfica. Ele possibilitou chegar a algumas observações interessantes dos resultados, apresentadas a seguir.

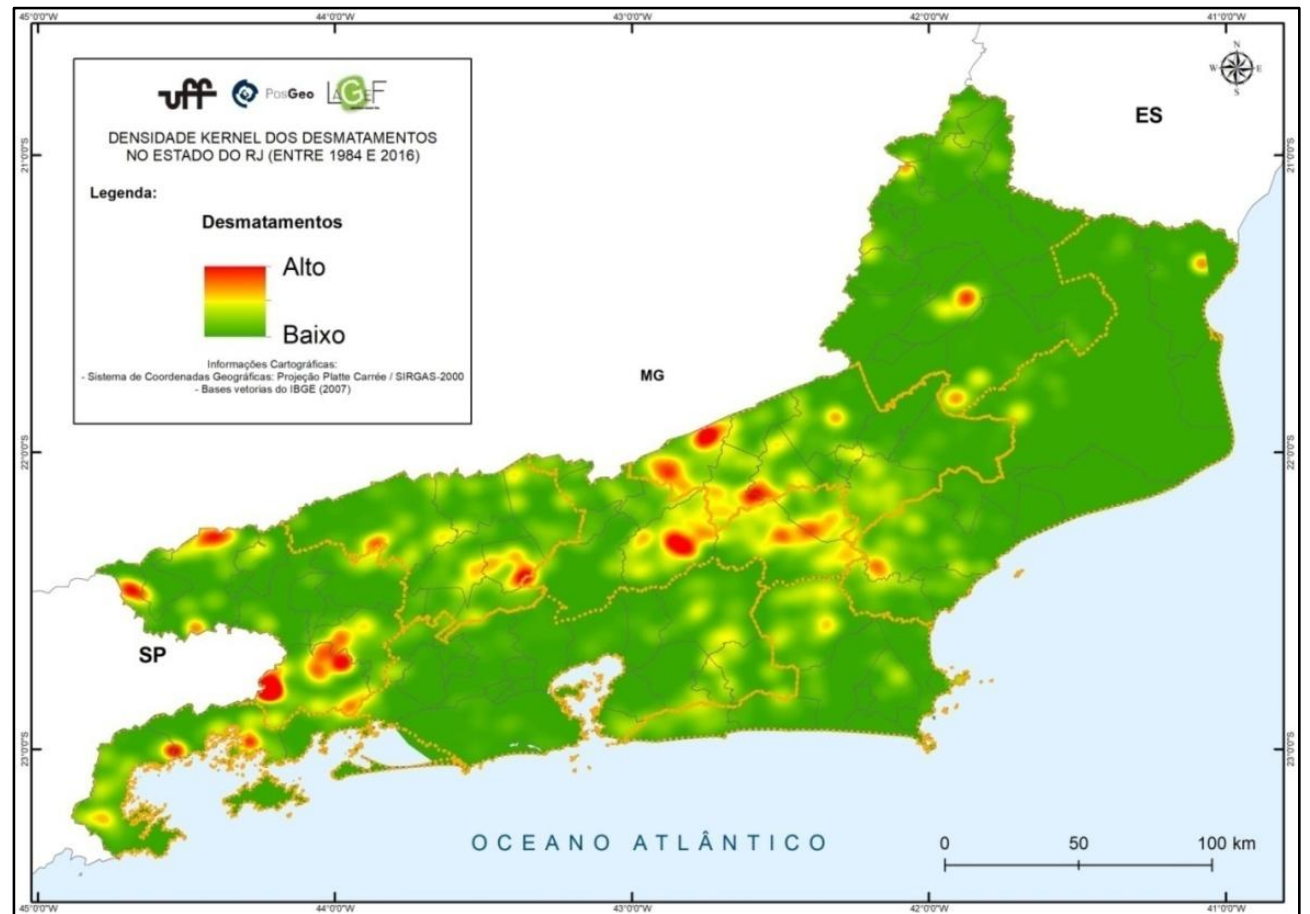

Figura 4: Densidade kernel das supressões florestais no estado do Rio de Janeiro, entre 1984 e 2016.

A densidade de ocorrência das supressões florestais chama muita atenção em duas áreas do estado. Primeiro, na Região Serrana, principalmente entre os municípios de Teresópolis, Nova Friburgo, Sumidouro e Sapucaia, áreas bem montanhosas, com escarpas e planaltos no reverso de escarpas. Nesta área as manchas de densidade chegam ao seu nível mais alto. Segundo, em menor escala, próximo à Região da Costa Verde, principalmente entre Angra dos Reis, Rio Claro e Piraí (Figura 4).

Quanto às recuperações, as principais manchas estão na fronteira entre as Regiões Serrana e Turística dos Lagos, na borda entre as montanhas serranas e as planícies litorâneas, nos municípios de Nova Friburgo, Casimiro de Abreu, Rio das Ostras e a parte montanhosa de Macaé. Áreas estas que fazem parte de importantes bacias hidrográficas, como as do Rio São João e do Rio Macaé (Figura 5). 


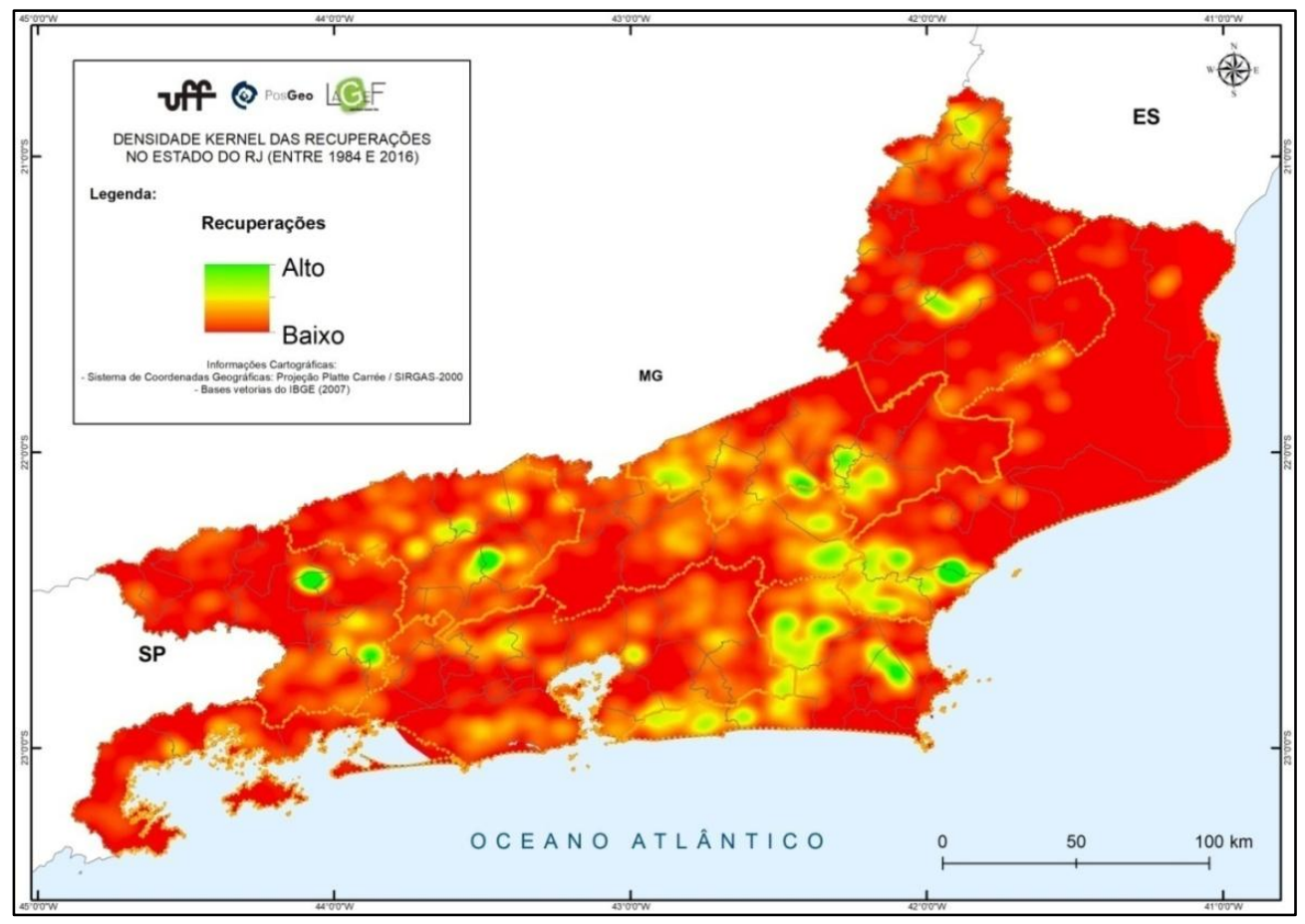

Figura 5: Densidade kernel das recuperações florestais no estado do Rio de Janeiro, entre 1984 e 2016. 3.3 Características das trajetórias florestais do estado do Rio de Janeiro

As supressões florestais são em sua maioria antigas, com aproximadamente $60 \%$ das ocorrências durante a década de 1980. Quanto à duração destas supressões há dois picos de ocorrência: um com perda vegetacional abrupta, entre 1 e 3 anos; e um com degradação contínua da paisagem, com perda sucessiva entre 29 e 32 anos de duração; representando $25 \%$ e $40 \%$ do total, respectivamente. Todas as perdas de floresta na área tiveram média magnitude no NDVI, entre 0.2 e 0.4 (Figura 5).

Em síntese, as perdas florestais foram mais antigas e graduais, ou seja, a degradação começou na década de 1980 e continuou por grande parte dos 32 anos de análise deste artigo. Estas supressões se concentraram no interior do estado, sobretudo na Região Serrana, famosa pelas recorrentes catástrofes ambientais de deslizamentos e enchentes.

As recuperações florestais ocorreram em dois principais momentos do período de análise: entre 1985 e 1986 foram 25\% do total e o ano de 2011, sozinho, representou 20\% dos ganhos florestais. Quase 60\% desses ganhos foram rápidos, ocorreram entre 3 e 6 anos de duração. Vales destacar também que $10 \%$ dos ganhos foram graduais, tiveram 31 anos de duração. Assim como as supressões, as recuperações também foram em sua maioria de média magnitude no NDVI (Figura 6).

Em síntese, as recuperações florestais no estado foram bem distribuídas quanto à sua ocorrência, parte antiga e parte mais recente. No entanto, em sua maioria, teve um comportamento mais abrupto, incondizente com o observado na natureza sem interferência antrópica. Há de se aprofundar os estudos quanto à estas recuperações, com campos e análises de imagens em alta resolução, pois tudo indica ser uma floresta plantada. Apenas um pouco mais de $10 \%$ destes ganhos florestais foram graduais, o que indica fortemente uma sucessão florestal. 


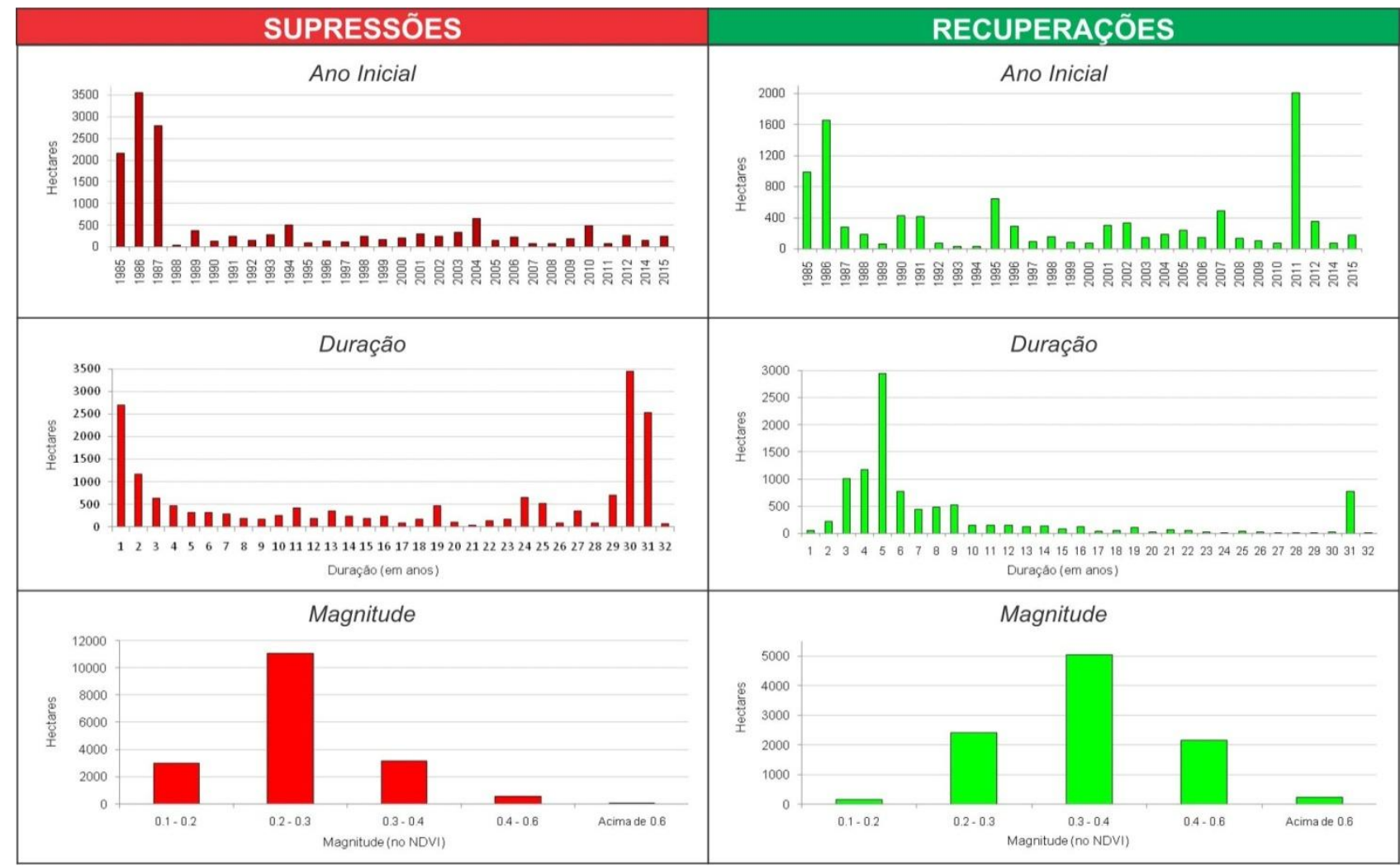

Figura 6: Resumo das características das trajetórias florestais (ano inicial, duração e magnitude).

\subsection{Validação das trajetórias}

Após a análise dos 1350 pontos de validação foi encontrado um índice Kappa de 0.53 no mapeamento de trajetórias, com uma exatidão global de $70 \%$, o que é considerado bom, de acordo com Landis e Koch (1977). Este resultado está entre o esperado para o algoritmo, visto que os trabalhos vistos na Literatura obtiveram uma exatidão global entre 65\% e 70\% (KENNEDY et al., 2010; GRIFITHS et al., 2012; KENNEDY et al., 2012; FRAGAL et al., 2016).

Analisando a matriz de confusão observa-se um acerto maior da classe de supressão em relação à de recuperação florestal. Em ambas o erro de omissão foi maior, ou seja, foram superestimadas em relação ao real. Já a classe de invariantes, apesar de ótimos resultados, foi subestimada, pois obteve alto erro de comissão (Tabela 3).

Numa análise geral dos erros, identificou-se três fontes dos mesmos: (1) proveniente das máscaras construídas no pré-processamento; (2) devido à elevada fragmentação florestal em algumas áreas; (3) imagens em períodos sazonais diferentes. Especificamente a Região IX obteve os maiores erros de omissão, prejudicando os dados gerais do estado. Acredita-se que a vegetação semidecidual e decidual desta região, associada à imagens de épocas distintas tenham confundido o algoritmo, que identificou uma mudança, onde na verdade era apenas diferença de umidade entre as estações seca e úmida, sendo portanto, uma área invariante.

Tabela 3: Matriz de confusão de validação da classicação de trajetórias florestais.

\begin{tabular}{lccccc}
\hline Classificação x Verdade & Supressão & Recuperação & Invariante & TOTAL & Omissão (\%) \\
\hline Supressão & $\mathbf{2 7 1}$ & 45 & 134 & 450 & 39 \\
Recuperação & 82 & $\mathbf{2 4 1}$ & 127 & 450 & 46 \\
Invariante & 17 & 22 & $\mathbf{4 1 1}$ & 450 & 8 \\
Comissão (\%) & 27 & 22 & 39 & Exatidão global: & $70 \%$ \\
\hline
\end{tabular}

\subsection{Mapa Sintese das Trajetórias}

A associação entre os níveis possibilitou sintetizar todas as trajetórias e suas características em 5 classes que compõe o mapa síntese das trajetórias florestais do estado do Rio de Janeiro (Figura 7). Observa-se no geral uma predominância de degradação no estado, sendo que no interior as supressões são antigas e graduais. Na Região IX, extremo norte do estado, também no interior, elas são antigas e abruptas. A região 
mais populosa, a Urbano-Industrial (IV), se manteve estável com perdas florestais recentes e recuperações antigas, ambos abruptos. A Região VIII, também estável, com supressões e recuperações antigas e abruptas. Interessante notar que nas áreas próximas a grandes centros industriais, entre a metrópole do Rio de Janeiro e a Região do Petróleo e Gás, houve mais supressões abruptas, enquanto que no interior do estado este fenômeno foi mais gradual. Já no litoral do estado observou-se características diferentes de trajetórias, a única região com tendência de recuperação foi a VII (Turística dos Lagos), com ganhos florestais antigos e de média duração. Em todas as classes síntese a magnitude predominante dos fenômenos foi a média.

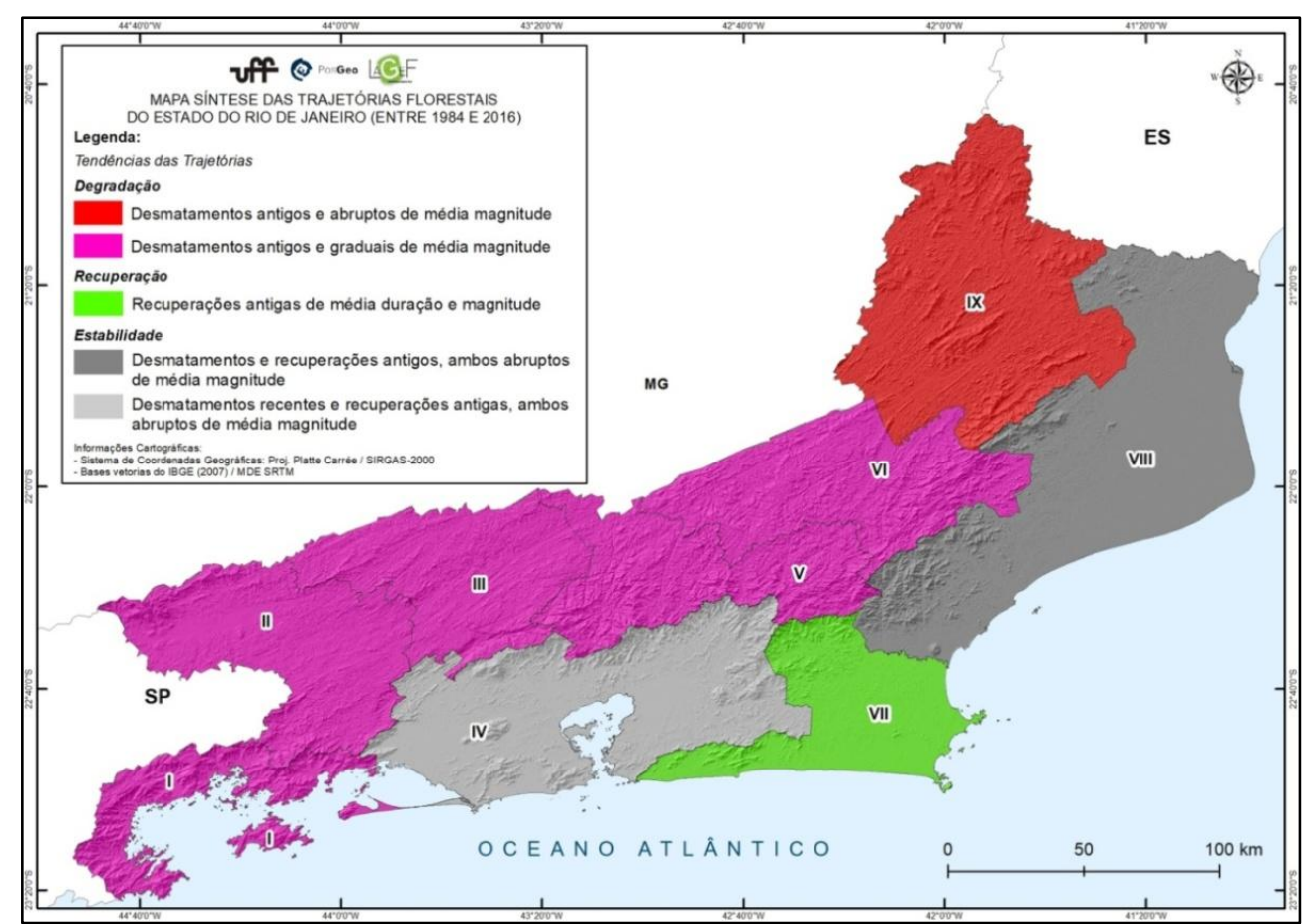

Figura 7: Síntese das trajetórias florestais do estado do Rio de Janeiro (entre 1984 e 2016), com suas respectivas tendências de paisagem. Regiões: I - Turística da Costa Verde; II - Industrial do Médio Paraíba; III - Turístico-Cultural do Médio Paraíba; IV - Urbana-Industrial; V - Serrana de Economia Diversificada; VI - Serrana de Economia Agropecuária; VII - Turística dos Lagos; VIII - Petróleo e Gás Natural; IX Agropecuária do Pomba, Muriaé e Itabapoana.

\section{Considerações Finais}

De uma maneira geral, a metodologia mostrou-se sólida e condizente com os produtos gerados. As validações ao final de cada etapa apresentaram bons resultados, ratificando a eficiência das várias abordagens utilizadas em conjunto neste trabalho.

O pacote de algoritmos LandTrendr foi muito eficiente em detectar trajetórias florestais, sejam de distúrbios ou recuperações. Seu método automatizado de segmentação temporal, pixel a pixel (porém observando uma janela de pixels vizinhos) e baseado na observação de uma série temporal e suas curvas espectrais, foi capaz de detectar mudanças florestais, mesmo as mais sutis, com um baixo custo e em um curto período de tempo. Mesmo os maiores recortes da área de estudo levaram menos de 24 horas de processamento para se obter dados de trajetórias espectro-temporais e suas características.

A predominância de supressões florestais era esperada devido ao longo histórico deste fenômeno no estado do Rio de Janeiro, desde os antigos ciclos econômicos até as atuais pressões provocadas pelo inchaço populacional e pelo mercado imobiliário. Foi interessante notar a diferença de características entre perdas florestais no interior do estado e nas áreas mais próximas ao litoral. A predominância de recuperações na Região Turística dos Lagos foi uma grata supresa, visto que é uma área em que o mercado imobiliátio cresceu muito nos últimos 30 anos.

Os mapeamentos das características de trajetórias oferecidas pelo algoritmo LandTrendr (ano inicial, duração e magnitude) se mostraram úteis para auxiliar no entendimento destes fenômenos, além de condizentes com as particularidades de cada região. Condensar estes dados no mapa síntese foi uma boa decisão, pois acredita-se que a legenda sintética deste representa o histórico espectro-temporal das supressões e recuperações, além da tendência de trajetórias em cada região do estado. 
Acredita-se que a metodologia desenvolvida e validada neste artigo possui grande potencial para a detecção de mudanças florestais, apresentando resultados sólidos e condizentes com a realidade da área de estudo e dos materiais utilizados, podendo ser replicada para outras áreas, com outras realidades. Assim, fica a necessidade de estudos mais aprofundados com relação a essa temática, que possam, a partir das contribuições do presente trabalho desenvolver futuras pesquisas analisando, elaborando e propondo medidas que ajudem a compreender as trajetórias evolutivas florestais.

\section{Agradecimentos}

Os autores agradecem ao Laboratório de Geografia Física (LAGEF), da Universidade Federal Fluminense (UFF); ao Conselho Nacional de Desenvolvimento Científico e Tecnológico (CNPq) e a Coordenação de Aperfeiçoamento de Pessoal de Nível Superior (Capes) pelo apoio a esta pesquisa.

\section{Referências}

BANSKOTA, A.; KAYASTHA, N.; FALKOWSKI, M. J.; WULDER, M. A.; FROESE, R. E.; WHITE, J. C. Forest Monitoring Using Landsat Time Series Data: A Review. Canadian Journal of Remote Sensing, v. 40 n. 5, pp.362-384, 2014.

CÂMARA, G.; MACIEL, A.; MAUS, N.; VINHAS, L.; SANCHEZ, A. Using dynamic geospatial ontologies to support information extraction from big Earth observation data sets. GIScience Conference, 2016.

CHEN, G.; HAY, G. J.; CARVALHO, L. M. T.; WULDER, M. A. Object based change detection. International Journal of Remote Sensing, v. 33, n. 14, pp. 4434-4457, 2012.

COHEN, W. B.; YANG, Z.; KENNEDY, R. E. (2010) Detecting trends in forest disturbance and recovery using yearly Landsat time series: 2. TimeSync - Tools for calibration and validation. Remote Sensing of Environment, v. 114, pp. 2911-2924.

CRONEMBERGER, F. M. Paisagens da Serra do Mar: uma análise geoecológica da dinâmica da paisagem. Tese de Doutorado em Geografia, UFF. Niterói, RJ. 159 p, 2014.

CRUZ, C. B. M.; VICENS, R. S.; SEABRA, S.; REIS R. B.; FABER, O. A.; ARNAUT, P. K. E.; ARAÚJO, M. Classificação orientada a objetos no mapeamento dos remanescentes da cobertura vegetal do bioma Mata Atlântica, na escala 1:250.000. Anais do XIII Simpósio Brasileiro de Sensoriamento Remoto. Florianópolis, SC. INPE, pp. 5691-5698, 2007.

FERNANDES, P. J. F.; VICENS, R. S.; FURTADO, L. F. A. Modelo automático de normalização radiométrica de série multitemporal Landsat-5 usando pontos pseudoinvariantes, PIF. Revista Brasileira de Cartografia, v. 69, n. 02, pp. 241-251, 2017.

FERRAZ, D. P. G. B. Dinâmica espaço-temporal da produção de silvicultura no estado do Rio de Janeiro entre 1985 e 2014. Dissertação de Mestrado em Geografia, UFF. Niterói, RJ. 83 p, 2017.

FRAGAL, E. H. Reconstrução histórica de mudanças na cobertura florestal em várzeas do baixo Amazonas utilizando o algoritmo Landtrendr. Dissertação de Mestrado em Sensoriamento Remoto, Instituto Nacional de Pesquisas Espaciais (INPE), São José dos Campos, SP. 126 p, 2015.

FRAGAL, E. H.; SILVA, T. S. F.; NOVO, E. M. L. M. Reconstructing historical forest cover change in the Lower Amazon floodplains using the LandTrendr algorithm. Acta Amazonica, v. 46, n. 1, pp. 13-24, 2016.

GRIFFITHS, P; KUEMMERLE, T; KENNEDY, R. E; ABRUDAN, I. V; KNORN, J; HOSTERT, P. Using annual time-series of Landsat images to assess the effects of forest restitution in post-socialist Romania. Remote Sensing of Enviroment, v. 118, pp. 199-214, 2012.

JENSEN, J. R. Sensoriamento Remoto do Ambiente. Uma Perspectiva em Recursos Terrestres. Ed. Parentese, São José dos Campos, SP. 598 p., 2009.

JIANYA, G.; HAIGANG, S.; GUORUI, M.; QIMING, Z. A Review of Multi-Temporal Remote Sensing Data Change Detection Algorithms. The International Archives of the Photogrammetry, Remote Sensing and Spatial Information Sciences. Vol. XXXVII. Part B7. Beijing, China, 2008.

KENNEDY, R. E.; ANDRÉFOUËT, S.; COHEN, W. B.; GÓMEZ, C.; GRIFFITHS, P.; HAIS, M.; HEALEY, S. P.; HELMER, E. H.; HOSTERT, P.; LYONS, M. B.; MEIGS, G. W.; PFLUGMACHER, D.; 
PHIN, S. R.; POWELL, S. L.; SCARTH, P.; SEN, S.; SCHROEDER, T. A.; SCHNEIDER, A.; SONNENSCHEIN, R.; VOGELMANN, J. E.; WULDER, M .A.; ZHU, Z. Bringing an ecological view of change to Landsat-based remote sensing. Frontiers in Ecology and Environment, v. 12 (6), pp. 339-346, 2014.

KENNEDY, R. E.; COHEN, W. B.; SCHROEDER, T. A. Trajectory-based change detection for automated characterization of forest disturbance dynamics. Remote Sensing of Environment, v. 110, pp. 370-386, 2007.

KENNEDY, R. E.; YANG, Z.; COHEN, W. B. Detecting trends in forest disturbance and recovery using yearly Landsat time series: 1. LandTrendr - Temporal segmentation algorithms. Remote Sensing of Environment, v. 114, pp. 2897-2910, 2010.

KENNEDY, R. E.; YANG, Z.; COHEN, W. B.; PFAFF, E.; BRAATEN, J.; NELSON, P. Spatial and temporal patterns of forest disturbance and regrowth within the area of the Northwest Forest. Remote Sensing of Environment, v. 122, pp. 117-133, 2012.

KENNEDY, R. E.; YANG, Z.; GORELICK, N.; BRAATEN, J.; CAVALCANTE, L.; COHEN, W. B.; HEALEY, S. Implementation of the LandTrendr Algorithm on Google Earth Engine. Remote Sensing, v. 10, n. 691, pp. 1-10, 2018.

LANDIS, J. R.; KOCH, G. G. The measurement of observer agreement for categorical data. Biometrics, v. 33, pp.159-174, 1977.

LU, D.; LI, G.; MORAN, E. Current situation and needs of change detection techniques. International Journal of Image and Data Fusion, v. 5, n. 1, pp. 13-38, 2014.

LU, D.; MAUSEL, P.; BRONDIZIO, E.; MORAN, E. Change detection techniques. International Journal of Remote Sensing, v. 25 n. 12, pp. 2365-2407, 2004.

MAUS, N.; CÂMARA, G.; CARTAXO, R.; SANCHEZ, A.; RAMOS, M.; QUEIROZ, G. R. A TimeWeighted Dynamic Time Warping Method for Land-Use and Land-Cover Mapping. IEEE Journal of Selected Topics in Applied Earth Observations and Remote Sensing, v. 9, n. 8, pp. 3729-3739, 2016.

PONZONI; F. J.; SHIMABUKURO, Y .E. Sensoriamento Remoto no Estudo da Vegetação. Ed. Parêntese, São José dos Campos, SP, 2 a edição. 144 p., 2009.

SARAÇA, C. E. S.; RAHY, I. S; SANTOS, M. A.; COSTA, M. B.; ALENCAR, R. S.; PERES, W. R. A propósito de uma nova regionalização para o Estado do Rio de Janeiro; p. 33-40. In: BERGALLO, H. G.; FIDALGO; E .C .C.; ROCHA, C. F. D.; UZÊDA; M. C.; COSTA, M .B.; ALVES, M. A. S.; VAN SLUYS, M.; SANTOS, M. A.; COSTA, T. C. C.; COZZOLINO, A. C. R. (ed.). Estratégias e ações para a conservação da biodiversidade no Estado do Rio de Janeiro. Rio de Janeiro, Instituto Biomas, 2007.

SCHOTT, J. R.; Salvaggio, C.; Volchok, W. J. Radiometric scene normalization using pseudoinvariant features. Remote Sensing of Environment, v. 26, pp. 1-16, 1988.

VELOSO, H. P.; RANGEL FILHO, A. L. R.; LIMA, J. C. A. Classificação da vegetação brasileira, adaptada a um sistema universal. Rio de Janeiro: IBGE/DERNA. 123 p., 1991.

VICENS, R. S.; CRONEMBERGER, F. M.; CRUZ, C. B. M. (2009) Análise multi-resolução e modelagem do conhecimento na diferenciação de fisionomias de florestas em remanescentes de Mata Atlântica no Estado do RJ. Anais do XIV Simpósio Brasileiro de Sensoriamento Remoto. Natal, RN. INPE, pp. 4519-4526, 2009.

WECKMÜLLER, R. Trajetórias evolutivas das coberturas florestais do estado do Rio de Janeiro utilizando os algoritmos LandTrendr. POSGEO, Tese de Doutorado em Geografia, UFF (disponível em: goo.gl/9KuJnK). Niterói, RJ. 123 p, 2018.

WECKMÜLLER, R.; VICENS, R. S. Método híbrido de detecção de mudanças: uma associação entre classificação baseada em objetos e baseada em pixels. Revista Brasileira de Cartografia, v. 68 n. 05, pp. 883-899, 2016.

WECKMÜLLER, R.; ZEBENDE, J. V. J.; VICENS, R. S. Escolha do melhor descritor para a detecção de trajetórias em florestas tropicais utilizando os algoritmos LandTrendr. Revista Continentes, v. 7 n. 13, pp. 68-84, 2018. 